03

\title{
Влияние массовой скорости на величину критического теплового потока при кипении деионизованной воды с недогревом в микроканальной системе охлаждения
}

\author{
(C) В.В. Кузнецов ${ }^{1,2}$, А.С. Шамирзаев ${ }^{1}$ \\ ${ }^{1}$ Институт теплофизики им. С.С. Кутателадзе СО РАН, \\ Новосибирск, Россия \\ ${ }^{2}$ Новосибирский государственный университет, Новосибирск, Россия \\ E-mail: vladkuz@itp.nsc.ru
}

Поступило в Редакцию 8 ноября 2017 г.

Экспериментально исследовано влияние массовой скорости на критический тепловой поток при кипении деионизованной воды, недогретой до температуры насыщения, в микроканальной системе охлаждения. Рабочий участок представлял собой медный блок с двумя микроканалами глубиной $0.36 \mathrm{~mm}$, шириной $2 \mathrm{~mm}$ и длиной $16 \mathrm{~mm}$. Массовая скорость изменялась от 100 до $600 \mathrm{~kg} /\left(\mathrm{m}^{2} \cdot \mathrm{s}\right)$, величина начального недогрева — от 30 до $70^{\circ} \mathrm{C}$. Обнаружено изменение характера кризиса кипения при увеличении массовой скорости и получено значительное увеличение критического теплового потока при полной конденсации пара в распределительной камере системы охлаждения.

DOI: 10.21883 /PJTF.2018.20.46809.17114

Достижения в области миниатюризации и повышения производительности электронных и лазерных систем обусловливают необходимость поиска эффективных методов отвода тепла от малоразмерных теплонапряженных объектов [1]. Тепловые потоки для компьютерных микропроцессоров уже сейчас превышают $100 \mathrm{~W} / \mathrm{cm}^{2}$ [2], в то время как для лазеров, СВЧ-приборов и радаров они достигают $1000 \mathrm{~W} / \mathrm{cm}^{2}$, поэтому традиционные способы воздушного охлаждения являются неэффективными. Применительно к тепловыделяющим устройствам малого размера двухфазные микроканальные системы охлаждения, которые реализуют скрытую теплоту фазового перехода, способны 
обеспечить отвод предельно высоких тепловых потоков при низких массовых скоростях и перепадах давления [2,3], в особенности при кипении жидкости, недогретой до температуры насыщения [4].

Существуют определенные ограничения в применении микроканальных систем охлаждения, обусловленные возникновением кризиса кипения [5], поэтому разработке методов увеличения критического теплового потока в последнее время уделяется повышенное внимание [2]. Среди процессов, приводящих к преждевременному кризису кипения, выделяется выброс пара из микроканалов в распределительную камеру системы охлаждения, приводящий к появлению сжимаемого объема и развитию пульсаций расхода жидкости $[5,6]$. Причиной выброса микроструй пара является кратковременный рост давления в микроканалах при возникновении пузырькового кипения, которое может существенно превышать давление в распределительной камере. Для подавления пульсаций расхода применяется сильное уменьшение проходного сечения на входе в микроканалы, но это приводит к значительному росту перепада давления. Это показывает, что термогидродинамические процессы в области сопряжения микроканалов и распределительной камеры могут оказывать определяющее влияние на величину критического теплового потока, но они являются крайне слабо изученными.

Целью настоящей работы является экспериментальное исследование влияния массовой скорости на величину критического теплового потока при вынужденной конвекции деионизованной воды, недогретой до температуры насыщения, в микроканальной системе охлаждения с распределительной камерой большого объема. Это создает условия для полной или частичной конденсации пара, выбрасываемого из микроканалов, и позволяет создавать многорядные системы охлаждения для интегрированных теплонапряженных узлов с повышенным критическим тепловым потоком.

Экспериментальный участок для исследования кризиса теплообмена при кипении в микроканальной системе охлаждения показан на рис. 1. Он представляет собой медный блок длиной $16 \mathrm{~mm}$ с двумя микроканалами шириной $2000 \mu \mathrm{m}$ и глубиной $360 \mu \mathrm{m}$, разделенными перегородкой толщиной $2000 \mu \mathrm{m}$, и верхней крышкой из полированной нержавеющей стали толщиной $2 \mathrm{~mm}$. Верхняя часть блока теплоизолирована фторопластовым кожухом. Микроканалы изготовлены микрофрезерованием с последующей обработкой шлифовальной бумагой, шероховатость

Письма в ЖТФ, 2018, том 44, вып. 20 


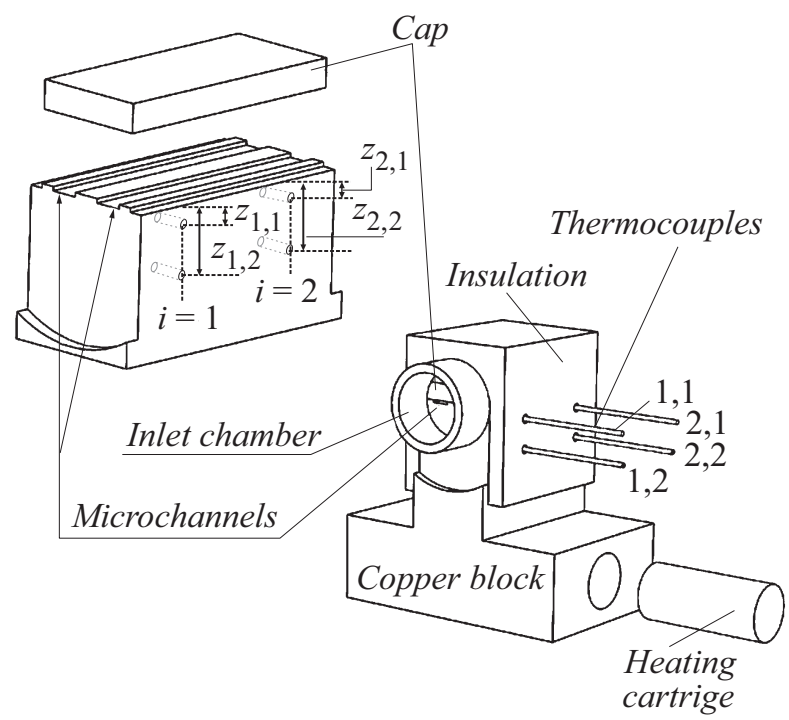

Рис. 1. Экспериментальный участок с микроканалами, термопарами и нагревателем.

поверхности $R_{a}$ равна $1.5 \mu \mathrm{m}$. Входная и выходная камеры экспериментального участка диаметром $8 \mathrm{~mm}$ и длиной $6 \mathrm{~mm}$ теплоизолированы от медного блока прокладками из паронита, в которых прорезаны щели с зазором $640 \mu \mathrm{m}$. Входная распределительная камера показана на рис. 1. Отношение поперечного сечения распределительной камеры к проходному сечению микроканалов равно 34.9. Это позволяет сохранять недогрев поступающей в камеру жидкости при конденсации пара, выбрасываемого из микроканалов. Температура потока измеряется на оси входной и выходной камер на расстоянии $6 \mathrm{~mm}$ от входа в микроканалы, отборники давления также расположены во входной и выходной камерах.

При проведении измерений деионизованная вода из бака-конденсатора подается дозирующим плунжерным насосом через гаситель пульсаций, термостат, фильтр, измеритель расхода и подогреватель в распределительную камеру экспериментального участка с микроканальной пластиной и затем направляется в бак-конденсатор. Массовая

6 Письма в ЖТФ, 2018, том 44, вып. 20 
скорость воды изменяется от 100 до $600 \mathrm{~kg} /\left(\mathrm{m}^{2} \cdot \mathrm{s}\right)$, величина начального недогрева жидкости до температуры насыщения изменяется от 30 до $70^{\circ} \mathrm{C}$. Измерение расхода жидкости производится расходомером Bronkhorst c неопределенностью $0.02 \mathrm{~g} / \mathrm{s}$. Давление в выходной камере поддерживается близким к атмосферному.

Тепловой поток к медному блоку подводится от нагревательного элемента, показанного на рис. 1. С двух сторон медного блока (на расстояниях от входа 3 и $13 \mathrm{~mm}$ ) установлены четыре пары заглубленных изолированных термопар диаметром $0.5 \mathrm{~mm}$. Расположение термопар на ближней стороне блока показано на рис. 1. Термопары расположены на расстояниях $z_{i, 1}=1.2 \mathrm{~mm}$ и $z_{i, 2}=5 \mathrm{~mm}( \pm 0.2 \mathrm{~mm})$ от основания микроканала и измеряют температуры $T_{i, 1}$ и $T_{i, 2}$. Индекс $i$ соответствует номеру термопары. Калибровка термопар проведена по платиновому термометру сопротивления в диапазоне температур от 16 до $340^{\circ} \mathrm{C}$, неопределенность измерения температуры составляет $0.2^{\circ} \mathrm{C}$.

Плотность теплового потока у основания микроканала

$$
q_{\text {eff }, i}=\lambda_{\mathrm{Cu}}\left(T_{i, 2}-T_{i, 1}\right) /\left(z_{i, 2}-z_{i, 1}\right)
$$

и температура стенки

$$
T_{w, i}=T_{i, 1}-q_{e f f, i} \cdot z_{i, 1} / \lambda_{\mathrm{Cu}}
$$

определяются по измеренному градиенту температуры в медном блоке и теплопроводности меди $\lambda_{\mathrm{cu}}$. Средний поток тепла на внутренней стенке микроканалов $q_{w}$ определяется отношением площади сечения медного блока $A_{e f f}$ к обогреваемой внутренней поверхности микроканалов

$$
A_{c h}=2(a+2 b) l,
$$

где $a$ и $b-$ ширина и высота микроканала, a $l-$ его длина. Дисбаланс количества тепла, выделяемого элементами с учетом теплопотерь, и тепла, передаваемого потоку, не превышает $4 \%$.

Для достижения кризиса теплообмена тепловой поток последовательно увеличивается при заданной массовой скорости. При установлении нового значения теплового потока производится непрерывное измерение температур в медном блоке, температуры жидкости во входной и выходной камерах, давления во входной камере и расхода до установления средних значений этих параметров. Переход к кризису

Письма в ЖТФ, 2018, том 44, вып. 20 

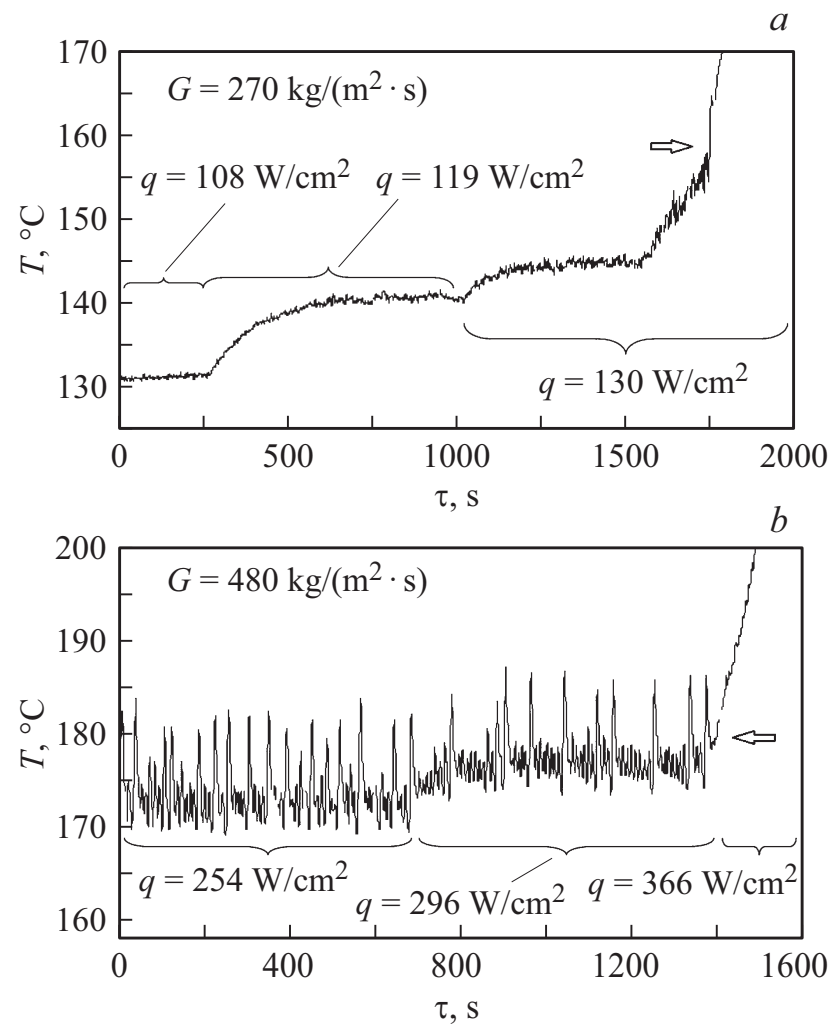

Pис. 2. Динамика температуры стенки во времени при последовательном увеличении теплового потока для термопары, расположенной на расстоянии $13 \mathrm{~mm}$ от входа. $G, \mathrm{~kg} /\left(\mathrm{m}^{2} \cdot \mathrm{s}\right): a-270, b-480$. Стрелкой показан момент возникновения кризиса теплообмена.

теплообмена соответствует быстрому росту температуры стенки или возникновению ее колебаний с амплитудой больше $30^{\circ} \mathrm{C}$.

На рис. 2 приведена динамика температуры стенки во времени при последовательном увеличении теплового потока, зарегистрированная на расстоянии $13 \mathrm{~mm}$ от входа для массовой скорости $G=270(a)$ и $480 \mathrm{~kg} /\left(\mathrm{m}^{2} \cdot \mathrm{s}\right)(b)$. При массовой скорости $270 \mathrm{~kg} /\left(\mathrm{m}^{2} \cdot \mathrm{s}\right)$ и тепловом потоке $108 \mathrm{~W} / \mathrm{cm}^{2}$ амплитуда колебаний температуры не превышает $0.5^{\circ} \mathrm{C}$

$6^{*}$ Письма в ЖТФ, 2018, том 44, вып. 20 

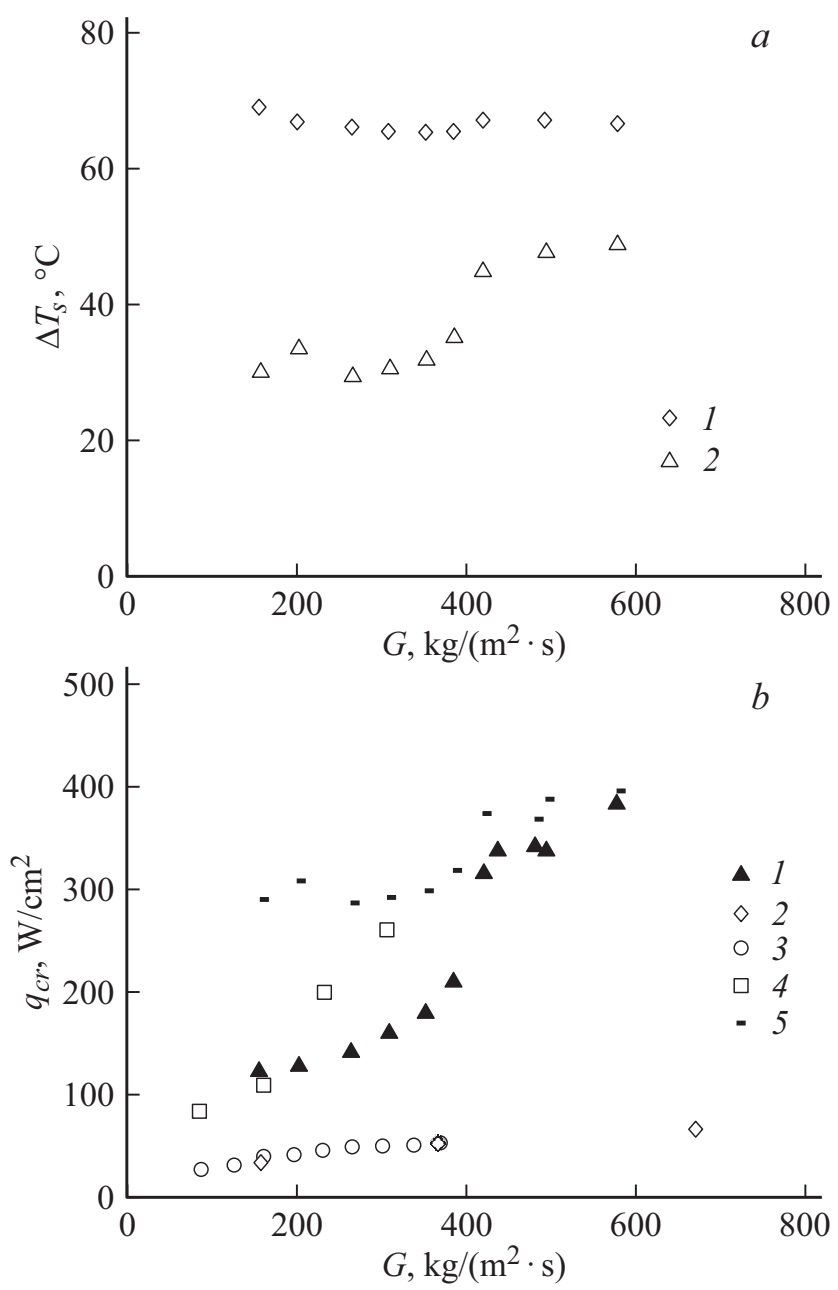

Рис. 3. Зависимости недогрева жидкости до температуры насыщения перед наступлением кризиса $(a)$ и критического потока $(b)$ от массовой скорости. a) 1 - недогрев поступающей воды, 2 - недогрев в распределительной камере; b) 1 - экспериментальные данные, 2 - данные [8], $3-[5], 4-[6], 5-$ расчет по данным [9]. 
и вызвана поверхностным кипением в условиях недогрева. Увеличение теплового потока до $119 \mathrm{~W} / \mathrm{cm}^{2}$ вызывает увеличение амплитуды колебаний температуры стенки из-за выброса пара из микроканалов и его конденсации, о чем свидетельствует рост температуры в распределительной камере. При увеличении плотности теплового потока до $130 \mathrm{~W} / \mathrm{cm}^{2}$ амплитуда колебаний и среднее значение температуры стенки нарастают во времени. В момент времени, обозначенный стрелкой на рис. 2,a, происходит быстрый рост температуры, давления и падение расхода. Это свидетельствует о развитии преждевременного кризиса теплообмена, вызванного образованием нарастающего объема пара в распределительной камере вследствие его неполной конденсации из-за повышения температуры жидкости выше предельного значения. Подобный характер развития преждевременного кризиса теплообмена представлен в [5].

На рис. 2, $b$ приведена динамика температуры стенки во времени для массовой скорости $480 \mathrm{~kg} /\left(\mathrm{m}^{2} \cdot \mathrm{s}\right)$. Как видно, при увеличении массовой скорости достижимая плотность теплового потока значительно увеличивается и динамика температуры во времени изменяется. В докризисном режиме теплообмена наблюдаются установившиеся колебания температуры, достигающие $8^{\circ} \mathrm{C}$, и кризис теплообмена возникает при тепловом потоке $366 \mathrm{~W} / \mathrm{cm}^{2}$. Подобное изменение динамики температуры стенки во времени в условиях недогрева ранее не наблюдалось и вызвано переходом к пульсирующей полной конденсации пара из-за сохранения достаточного недогрева в распределительной камере.

На рис. 3, а точками 1 показана зависимость недогрева жидкости, поступающей в распределительную камеру, $\Delta T_{s}=T_{s}-T_{f}$ от массовой скорости. Температура насыщения $T_{s}$ определена по давлению в камере. Точки 2 показывают недогрев жидкости в распределительной камере, который уменьшается из-за конденсации пара. Как видно, неполная конденсация пара и его накопление в камере происходят при установлении недогрева, близкого к $30^{\circ} \mathrm{C}$. Это значение соответствует границе устойчивости струи пара, истекающей в большой объем из сопла диаметром $1 \mathrm{~mm}$ [7]. При величине недогрева в распределительной камере больше $50^{\circ} \mathrm{C}$ конденсация истекающего пара является полной и критический тепловой поток значительно возрастает.

На рис. 3, $b$ показана зависимость критического теплового потока от массовой скорости. Точки 1 показывают экспериментальные данные, точки 2 и 3 - данные [8] и [5] для теплообменников с малым объемом

Письма в ЖТФ, 2018, том 44, вып. 20 
распределительной камеры, точки 4 - данные [6] для теплообменника с сужением входного проходного сечения в 10 раз. Как видно, даже неполная конденсация пара в распределительной камере приводит к существенному увеличению критического теплового потока, который значительно возрастает при полной конденсации пара и достигает значений, соответствующих расчету по данным [9] для кризиса кипения первого рода. Отметим, что при близких критических тепловых потоках отношение перепада давления на длине теплообменника перед кризисом к начальному перепаду давления не превышает пяти, в то время как для теплообменника с изменением входного проходного сечения оно достигает сорока [6].

Полученные данные показывают изменение механизма кризиса теплообмена при росте массовой скорости для кипения недогретой деионизованной воды и определяют условия значительного роста критического теплового потока при увеличении полноты конденсации пара, выбрасываемого из микроканалов. Они определяют эффективность применения микроканальных систем охлаждения для компактных многорядных систем охлаждения интегрированных теплонапряженных узлов, в том числе микропроцессоров с трехмерной архитектурой.

Исследование выполнено в ИТ СО РАН за счет гранта Российского научного фонда (проект № 16-19-10519).

\section{Список литературы}

[1] Чиннов Е.А., Роньшин Ф.В., Кабов О.А. // Письма в ЖТФ. 2015. Т. 41. В. 17. C. $1-8$.

[2] Mudawar I. // J. Therm. Sci. Eng. Appl. 2013. V. 5. N 2. P. 021012.

[3] Kuznetsov V.V., Shamirzaev A.S. // J. Eng. Thermophys. 2017. V. 26. N 2. P. 146153.

[4] Zeigarnik Y.A., Privalov N.P., Klimov A.I. // Thermal Eng. 1981. V. 28. N 1. P. 40-43.

[5] $Q u$ W., Mudawar I. // Int. J. Heat Mass Transfer. 2004. V. 47. N 10-11. P. 20452059.

[6] Kosar A., Kuo C.-J., Peles Y. // J. Heat Transfer. 2006. V. 128. N 3. P. 251-260.

[7] Cumo M., Farello G.E., Ferrari G.E. // Proc. of the 6th Int. Heat Transfer Conf. Toronto, 1978. V. 5. P. 101-106.

[8] Steinke M.E., Kandlikar S.G. // J. Heat Transfer. 2004. V. 126. N 4. P. 518-526.

[9] Кутателадзе С.С. Основы теории теплообмена. М.: Атомиздат, 1979.416 с.

Письма в ЖТФ, 2018, том 44, вып. 20 